IP Periodica Polytechnica Civil Engineering

60(2), pp. 247,255, 2016

DOI: $10.3311 /$ PPci.8192

Creative Commons Attribution (1)

RESEARCH ARTICLE

\section{A Numerical Method for Eigensolution of Near-Regular Structural and Mechanical Systems}

\author{
Iman Shojaei, Ali Kaveh, Hossein Rahami
}

Received 30-04-2015, revised 26-06-2015, accepted 30-11-2015

\begin{abstract}
In this paper a numerical method is developed to find the eigenvalues of the Laplacian matrix for near-regular graph models. Considering the similarity between the pattern of the Laplacian matrix of a graph and the stiffness matrix of a structure, the method can be used for the eigensolution of structural/mechanical systems. Previously, using graph product rules algorithms for the swift solution of the equation $\mathbf{F}=\mathbf{K} \Delta$ in structural/mechanical systems were developed. In this study, using a purposeful ordering along with partitioning the Laplacian/stiffness matrix, a decomposable pattern is achieved. The decomposed parts are solved using combined graph product rules and numerical solutions. While the eigensolution of regular patterns has been traditionally considered as an approximation for near-regular patterns, here the solution of the regular pattern is utilized as an appropriate initial starting point for the solution of near-regular pattern. Furthermore, solving the decomposed regular part using graph product rules reduces the computational complexity of the method.
\end{abstract}

\section{Keywords}

Regular structures · Near-regular structures · Eigensolution . Graph products $\cdot$ Laplacian matrix

\section{Iman Shojaei}

Department of Biomedical Engineering, University of Kentucky, Lexington, KY 40506, USA

\section{Ali Kaveh}

Centre of Excellence for Fundamental Studies in Structural Engineering Iran University of Science and Technology, Narmak, Tehran, 1684613114, Iran e-mail: alikaveh@iust.ac.ir

\section{Hossein Rahami}

Engineering Optimization Research Group, College of Engineering; School of Engineering Science, College of Engineering, University of Tehran, Tehran, Keshavarz Blvd., No. 37, Ghods St., Iran

\section{Introduction}

Although the advent of powerful computers have smoothened the way for the swift computations structural/mechanical systems, the analysis of complicated systems are still laborious and time-consuming. Many algorithms for the solutions of largescale structural/mechanical systems have been developed over the past three decades. These algorithms have mainly aimed to find efficient solutions for the structural governing equations (i.e. $\mathbf{F}=\mathbf{K} \Delta$ ) and/or eigensolution of a system (i.e. frequencies of free vibration). However, due to the variety of systems and lack of general patterns, most algorithms are limited to partial applications. The most successful advancements were achieved in the solution of symmetric and regular patterns wherein linear algebra, graph products, group theoretical method, U-transformation etc. were employed to divide complicated large problems into sub-systems and solve the smaller parts with less computational complexity and then combine the solutions (i.e. divide and conquer methods) [1-7].

There are various structural/mechanical systems with geometries close to those of regular structures, but not satisfying the required mathematical conditions to be considered as regular. A model is called regular if it can be considered as the product of two or three graphs [1]. A near regular model consists of a regular submodel with limited number of members and/or nodes being added or removed. Recent efforts have been devoted to realize, classify and solve these near-regular patterns efficiently through the available solution of the regular part $[8-12]$. While several flexibility and stiffness methods, and finite difference and finite element formulations were developed to solve the final governing equation $\mathbf{F}=\mathbf{K} \Delta$, less success were achieved for the eigensolution of the near-regular systems [8-12]. Compared to the solution of $\mathbf{F}=\mathbf{K} \Delta$, eigenvalue problems are more sensitive to the algebraic manipulations. This often leads to matrices with general patterns that cannot usually be solved using specific efficient solvers.

In this paper a numerical algorithm is presented for obtaining the eigenvalues of near-regular structures. In this method utilizing the decomposition of block matrices, a determinant equation is obtained. This equation includes separate submatrices corre- 
sponding to the regular and irregular parts of the system. The equation is then solved with a numerical method. The swiftness of the solution corresponds to: 1) Appropriate initial starting point of the solution (i.e. the eigenvalues of the regular structure) and 2) Efficient solution of the separated matrix (corresponding to the regular system), in each iteration, using graph product rules.

\section{Eigenvalues for the regular graph products}

Sufficient conditions for converting Hermitian matrices $\mathbf{A}_{1}$ and $\mathbf{A}_{2}$ into upper triangular ones using one orthogonal matrix are [11, 13, 14]:

$$
\mathbf{A}_{1} \mathbf{A}_{2}=\mathbf{A}_{2} \mathbf{A}_{1} \text { or } \mathbf{A}_{1}^{2}=\mathbf{A}_{2}^{2}
$$

Consider the matrix $\mathbf{M}$ to be the sum of two Kronecker products:

$$
\mathbf{M}=\mathbf{A}_{1} \otimes \mathbf{B}_{1}+\mathbf{A}_{2} \otimes \mathbf{B}_{2}
$$

If one of the conditions in Eq. (1) holds, the eigenvalues of the block matrix $\mathbf{M}$ are obtained as follows:

$$
\lambda_{\mathbf{M}}=\bigcup_{i=1}^{n} \operatorname{eig}\left(\mathbf{M}_{i}\right) ; \mathbf{M}_{i}=\lambda_{i}\left(\mathbf{A}_{1}\right) \mathbf{B}_{1}+\lambda_{i}\left(\mathbf{A}_{2}\right) \mathbf{B}_{2}
$$

In this equation, the dimension of matrices $\mathbf{A}_{1}$ and $\mathbf{A}_{2}$ is equal to $n$, and the dimension of matrices $\mathbf{B}_{1}$ and $\mathbf{B}_{2}$ is equal to $m$.

In structural engineering block diagonal matrices, especially tri-block diagonal matrices, are of great importance. Some regular forms of these matrices were solved previously [13, 15]. The following two forms correspond to the stiffness matrices of regular structures:

$$
\begin{aligned}
\mathbf{F}_{n}\left(\mathbf{A}_{m}, \mathbf{B}_{m}, \mathbf{C}_{m}\right) & =\left[\begin{array}{ccccc}
\mathbf{A}_{m} & \mathbf{B}_{m} & & & \\
\mathbf{B}_{m} & \mathbf{C}_{m} & \mathbf{B}_{m} & & \\
& \mathbf{B}_{m} & \ddots & \ddots & \\
& & \ddots & \mathbf{C}_{m} & \mathbf{B}_{m} \\
& & & \mathbf{B}_{m} & \mathbf{A}_{m}
\end{array}\right] ; \\
\mathbf{G}_{n}\left(\mathbf{A}_{m}, \mathbf{B}_{m}, \mathbf{C}_{m}\right) & =\left[\begin{array}{lllll}
\mathbf{A}_{m} & \mathbf{B}_{m} & & & \mathbf{B}_{m} \\
\mathbf{B}_{m} & \mathbf{C}_{m} & \mathbf{B}_{m} & & \\
& \mathbf{B}_{m} & \ddots & \ddots & \\
& & \ddots & \mathbf{C}_{m} & \mathbf{B}_{m} \\
\mathbf{B}_{m} & & & \mathbf{B}_{m} & \mathbf{A}_{m}
\end{array}\right]
\end{aligned}
$$

The solved patterns consist of the forms $\mathbf{G}_{n}\left(\mathbf{A}_{m}, \mathbf{B}_{m}, \mathbf{A}_{m}\right)$, $\mathbf{F}_{n}\left(\mathbf{A}_{m}, \mathbf{B}_{m}, \mathbf{A}_{m}\right)$ and the form $\mathbf{F}_{n}\left(\mathbf{A}_{m}, \mathbf{B}_{m}, \mathbf{C}_{m}\right)$ with the condition $\mathbf{A}_{m}-\mathbf{B}_{m}=\mathbf{C}_{m}$. While the forms $\mathbf{G}_{n}\left(\mathbf{A}_{m}, \mathbf{B}_{m}, \mathbf{C}_{m}\right)$ and the form $\mathbf{F}_{n}\left(\mathbf{A}_{m}, \mathbf{B}_{m}, \mathbf{C}_{m}\right)$ with the condition $\mathbf{A}_{m}-\mathbf{B}_{m} \neq \mathbf{C}_{m}$ are not completely regular to be swiftly solved.

\section{Decomposition of partitioned block matrices}

This section is devoted to some techniques of partitioned block matrices. Manipulating a partitioned matrix is a basic and helpful approach in matrix analysis. The applied methods in partitioned matrices are similar to those of ordinary numerical matrices in some ways. Consider a $2 \times 2$ matrix as

$$
\mathbf{A}=\left[\begin{array}{ll}
a & b \\
c & d
\end{array}\right], a, b, c, d \in C
$$

Using a basic row operation, the second row of the matrix multiplied by 2 can be added to the first row so that the determinant of the matrix does not change:

$$
\left[\begin{array}{ll}
1 & 2 \\
0 & 1
\end{array}\right]\left[\begin{array}{ll}
a & b \\
c & d
\end{array}\right]=\left[\begin{array}{cc}
a+2 c & b+2 d \\
c & d
\end{array}\right]
$$

This row or column operation can be generalized to partitioned block matrices as

1 Interchanging two block rows or columns,

2 Multiplying a block row or column from the left or right by a matrix of proper size,

3 Multiplying a block row or column by a matrix and then adding it to another row or column.

It should be noted that for maintaining the symmetry of the matrix, these operations are performed simultaneously on rows and columns and therefore the determinant does not change.

Now using the above three basic rules one can convert a twoblock matrix into a two-block diagonal matrix so that the determinant does not change:

$$
\begin{aligned}
& {\left[\begin{array}{cc}
\mathbf{I}_{n} & \mathbf{0} \\
-\mathbf{M}_{21} \mathbf{M}_{11}^{-1} & \mathbf{I}_{m}
\end{array}\right]\left[\begin{array}{cc}
\mathbf{M}_{11} & \mathbf{M}_{12} \\
\mathbf{M}_{21} & \mathbf{M}_{22}
\end{array}\right]\left[\begin{array}{cc}
\mathbf{I}_{n} & -\mathbf{M}_{11}^{-1} \mathbf{M}_{12} \\
\mathbf{0} & \mathbf{I}_{m}
\end{array}\right]=} \\
& =\left[\begin{array}{cc}
\mathbf{M}_{11} & \mathbf{0} \\
\mathbf{0} & \mathbf{M}_{22}-\mathbf{M}_{21} \mathbf{M}_{11}^{-1} \mathbf{M}_{12}
\end{array}\right]
\end{aligned}
$$

where $\mathbf{M}_{11}$ is an invertible matrix of dimension $n$ and $\mathbf{M}_{22}$ is a matrix of dimension $m$

Consequently we will have

$$
\left|\begin{array}{ll}
\mathbf{M}_{11} & \mathbf{M}_{12} \\
\mathbf{M}_{21} & \mathbf{M}_{22}
\end{array}\right|=\operatorname{det} \mathbf{M}_{11} \operatorname{det}\left(\mathbf{M}_{22}-\mathbf{M}_{21} \mathbf{M}_{11}^{-1} \mathbf{M}_{12}\right)
$$

For finding the eigenvalues of the partitioned matrices, the matrix $\mathbf{M}_{11}$ is changed to the matrix $\mathbf{M}_{11}-\lambda \mathbf{I}_{n}$ and the matrix $\mathbf{M}_{22}$ is changed to the matrix $\mathbf{M}_{22}-\lambda \mathbf{I}_{m}$,

$$
\begin{aligned}
& \left|\begin{array}{cc}
\mathbf{M}_{11}-\lambda \mathbf{I}_{n} & \mathbf{M}_{12} \\
\mathbf{M}_{21} & \mathbf{M}_{22}-\lambda \mathbf{I}_{m}
\end{array}\right|=\operatorname{det}\left(\mathbf{M}_{11}-\lambda \mathbf{I}_{n}\right) \\
& \cdot \operatorname{det}\left(\mathbf{M}_{22}-\lambda \mathbf{I}_{m}-\mathbf{M}_{21}\left(\mathbf{M}_{11}-\lambda \mathbf{I}_{n}\right)^{-\mathbf{1}} \mathbf{M}_{12}\right)
\end{aligned}
$$


Equating this determinant to zero, results in:

$$
\operatorname{det}\left(\mathbf{M}_{11}-\lambda \mathbf{I}_{n}\right)=0
$$

and

$$
\operatorname{det}\left(\mathbf{M}_{22}-\lambda \mathbf{I}_{m}-\mathbf{M}_{21}\left(\mathbf{M}_{11}-\lambda \mathbf{I}_{n}\right)^{-\mathbf{1}} \mathbf{M}_{12}\right)=0
$$

Considering the condition of the decomposition where $\mathbf{M}_{11}$ in Eq. (8) or $\mathbf{M}_{11}-\lambda \mathbf{I}_{n}$ in Eq. (9) is invertible, Eq. (10) does not hold. Consequently, for finding the eigenvalues of the two-block matrix, only Eq. (11) should be solved.

As a simple example consider the following matrix

$$
\mathbf{M}=\left[\begin{array}{llll}
0.8147 & 0.6324 & 0.9575 & 0.9572 \\
0.9058 & 0.0975 & 0.9649 & 0.4854 \\
0.1270 & 0.2785 & 0.1576 & 0.8003 \\
0.9134 & 0.5469 & 0.9706 & 0.1419
\end{array}\right]
$$

Using Eq. (9), we will have

$$
\begin{aligned}
& \operatorname{det}\left(\left[\begin{array}{ll}
0.1576 & 0.8003 \\
0.9706 & 0.1419
\end{array}\right]-\lambda \mathbf{I}-\right. \\
& -\left[\begin{array}{ll}
0.1270 & 0.2785 \\
0.9134 & 0.5469
\end{array}\right]\left(\left[\begin{array}{ll}
0.8147 & 0.6324 \\
0.9058 & 0.0975
\end{array}\right]-\right. \\
& \left.-\lambda \mathbf{I})^{-1}\left[\begin{array}{ll}
0.9575 & 0.9572 \\
0.9649 & 0.4854
\end{array}\right]\right)=0
\end{aligned}
$$

The roots of this equation will be the eigenvalues of Matrix M

$$
\lambda_{\mathbf{M}}=\{2.4021,-0.0346,-0.7158,-0.4400\}
$$

Solving Eq. (9) results in finding the characteristic equation of the two-block matrix In general, finding the eigenvalues of matrices using their characteristic equation is not considered as a time-saving approach because both forming and solving the characteristic equation are difficult.

\section{Computational complexity of eigensolution of near-} regular graphs via solution of characteristic equation

Eigensolution of a matrix by solving the equation $\operatorname{det}(\mathbf{M}-\lambda \mathbf{I})=0$ includes forming the characteristic equation and then solving it by an iterative method. In addition to the difficulties inherent to the formation of characteristic equation, using an iterative method for calculating eigenvalues makes the method very complicated. However, when it comes to near-regular forms, the method changes to an efficient approach via the application of the features of the regular patterns.

Complexity theory has been well developed in numerical linear algebra. However, for eigenvalue problems where iterative methods are required, the complexity of algorithms cannot be easily obtained since the number of steps for achieving a desirable accuracy is not definite. Another problem is concerned with the initial starting point that is very important in many iterative algorithms which affects the number of iterations and consequently the computational complexity.

In this study, the difficulties of solving the equation $\operatorname{det}(\mathbf{M}-\lambda \mathbf{I})=0$, shown in Eq. (9) are smoothened for nearregular graphs. The method is carried out through

1 Removing the difficulty of inverting the matrix $\mathbf{M}_{11}-\lambda \mathbf{I}_{n}$, shown in Eq. (9).

2 Decreasing the complexity of the applied iterative method.

As it was mentioned, the matrices in this study are nearregular matrices composed of regular and irregular parts. Numbering the nodes of the graph (structure) is performed in such a way that the regular and irregular parts are separated. Therefore the Laplacian (stiffness) matrix will have a two-block matrix form $\mathbf{M}$ as shown in Eq. 6, where the block $\mathbf{M}_{11}$ represents the regular part and the block $\mathbf{M}_{22}$ represents the irregular part. Such an ordering can be seen in Fig. 1 for a near-regular graph

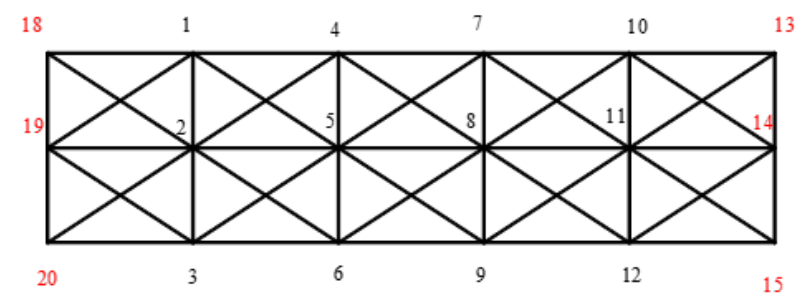

Fig. 1. A near-regular graph with a suitable ordering

Where the black numbers form the regular block $\mathbf{M}_{11}$ and the red ones form the irregular block $\mathbf{M}_{22}$. Previously, the inverse of the Laplacian matrix of regular graphs was obtained using its eigenpairs [15]:

$$
\begin{aligned}
& \mathbf{A} \mathbf{x}=\mathbf{b} \Rightarrow\{\varphi\}_{j}^{T} \mathbf{A}\{\varphi\}_{j} y_{j}=\lambda_{j} y_{j}=\{\varphi\}_{j}^{T} \mathbf{b} \\
& y_{j}=\frac{b_{j}}{\lambda_{j}} \Rightarrow\{x\}_{n}=\sum_{i=1}^{n}\{\varphi\}_{i} y_{i}=\sum_{i=1}^{n}\{\varphi\}_{i} \frac{b_{i}}{\lambda_{i}}=\sum_{i=1}^{n} \frac{\{\varphi\}_{i}\{\varphi\}_{i}^{T}}{\lambda_{i}} \mathbf{b}
\end{aligned}
$$

Where $\lambda_{i}$ and $\{\varphi\}_{i}$ are the eigenpairs of the matrix $\mathbf{A}$.

Since the block $\mathbf{M}_{11}$ is regular, $\mathbf{M}_{11}-\lambda \mathbf{I}_{n}$ is also regular. But in references [13-15], the eigenpairs and the inverted matrix were found for a regular matrix filled with numbers. Inverting the parametrical matrix $\mathbf{M}_{11}-\lambda \mathbf{I}_{n}$, solving the parametrical determinant and finding the characteristic equation is rather difficult. However, since the characteristic equation should be ultimately solved iteratively to find the eigenvalues, in the present study an approach is utilized in which the iterative method is applied at earlier stage. In other words, the iterative solution is directly applied to Eq. (9) without forming the characteristic equation. The advantage of applying the iterative solution in advance is that after making an initial guess for $\lambda$ in Eq. (9), the numerical matrix $\mathbf{M}_{11}-\lambda \mathbf{I}_{n}$ is quickly inverted using Eq. 10 and subsequently the determinant is solved. The determinant is 
of the dimension of the irregular part $\left(\mathbf{M}_{22}\right)$. Since in a nearregular graph we assume the irregular part to be small, the determinant is easily solved iteratively, until stopping criterion is satisfied. The iteration is performed using a bisection method to obtain all eigenvalues. Using strong initial guesses for the $\lambda_{i} \mathrm{~s}$ ensures the convergence of the solution. The Laplacian matrix for the graph shown in Fig. 1 can be written as

$$
\begin{aligned}
& \mathbf{M}=\left[\begin{array}{ll}
\mathbf{M}_{11} & \mathbf{M}_{12} \\
\mathbf{M}_{21} & \mathbf{M}_{22}
\end{array}\right] \\
& \left(\mathbf{M}_{11}\right)_{n m}=\mathbf{F}_{n}\left(\mathbf{C}_{m}, \mathbf{B}_{m}, \mathbf{C}_{m}\right)= \\
& \mathbf{I}_{\mathbf{n}} \otimes \mathbf{C}_{\mathbf{m}}+\mathbf{T}_{\mathbf{n}} \otimes \mathbf{B}_{\mathbf{m}}
\end{aligned}
$$

From Eq. 11 it can be seen that $\mathbf{T}_{n}=\mathbf{F}_{n}(0,1,0)$, and obviously $\mathbf{I}_{n} \mathbf{T}_{n}=\mathbf{T}_{n} \mathbf{I}_{n}$,i.e. the condition of Eq. (1) is satisfied and $\boldsymbol{M}$ has the property of being block diagonalized.

According to the definition of a near-regular structure, the regular part is much larger than the irregular part. In other words, in the computational complexity of a near-regular structure it is assumed that only the dimension of $\mathbf{M}_{11}$ (the regular part) approaches to infinity. Consequently, the initial guess is made using a matrix of the dimension of the matrix $\mathbf{M}$ but a pattern similar to the pattern of the regular matrix $\mathbf{M}_{11}$. Therefore, the following matrix is used for the initial guess

$$
\left(\mathbf{M}^{*}\right)_{l m}=\mathbf{F}_{l}\left(\mathbf{C}_{m}, \mathbf{B}_{m}, \mathbf{C}_{m}\right)
$$

Since the matrix $\mathbf{M}^{*}$ has a regular form, its eigenvalues are easily obtained. The eigenvalues of the matrix $\mathbf{M}^{*}$ are close to the eigenvalues of the matrix $\mathbf{M}$. The similarity becomes more obvious, when the regular part becomes larger.

Another way of finding the matrix $\mathbf{M}^{*}$ is to obtain the matrix $\mathbf{M}$ through a usual ordering as shown in Fig. 2. We will have

$$
\mathbf{M}_{n m}=\mathbf{F}_{n}\left(\mathbf{A}_{m}, \mathbf{B}_{m}, \mathbf{C}_{m}\right)
$$

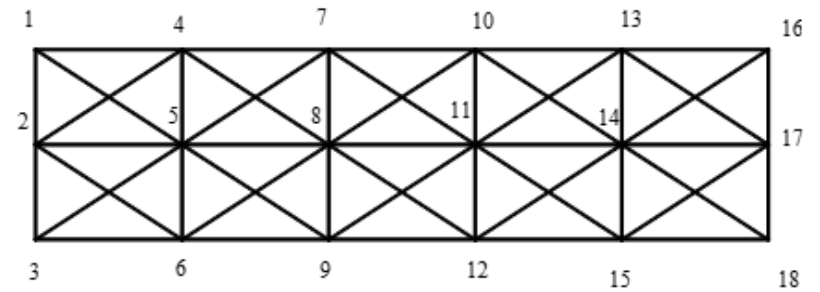

Fig. 2. A near-regular graph with a usual ordering

Since the graph follows the pattern of the strong Cartesian product, $\mathbf{A}_{m}-\mathbf{B}_{m} \neq \mathbf{C}_{m}$ and it is classified as a near-regular form. For converting the near-regular matrix $\mathbf{M}$ to a regular one, the block $\mathbf{A}_{m}$ is changed to the block $\mathbf{C}_{m}$. This conversion causes the regular matrix $\mathbf{M}^{*}$ to be obtained again. In Ref. [16] it is shown that addition of members around a graph, the condition $A_{m}-B_{m}=C_{m}$ holds and the eigenvaluses of the expanded graph are not much different with those of the graph before addition. Thus the eigenvalues of $\mathbf{M}$ and $\mathbf{M}^{*}$ are close to each other.

Up to now, a proper ordering was considered, the iterative method was applied in advance, the appropriate initial guess was made and the inverse of the matrix $\mathbf{M}_{11}-\lambda \mathbf{I}_{n}$ was found. Now, the complexity of the method is evaluated to show it is both measureable and less complicated. As it was mentioned, the computational complexity of many iterative methods is not measureable. The computational complexity of an iterative bisection method for finding the roots of the relationship $f(x)=0$ is measureable only in the case of finding a certain root in the interval $[a, b]$. However, while finding the multiple roots of an equation without having a sense of the domain of the roots, obtaining the complexity of the method is impossible. The problem gets worse when the order of the equation (the characteristic equation in our problems) grows. Growing the order of the equation is certain because in calculating the complexity of the method the order of the equation $(N)$ should approaches to infinity.

Now, it is shown why calculating the complexity of the method in the near-regular structures is possible. In a bisection method for finding roots in the interval $[a, b]$, the number of iterations $(n)$ can be obtained as follows:

$$
\frac{b-a}{2^{n+1}}<\varepsilon
$$

where $\varepsilon$ is the upper bound for the error of the answer. Eq. 14 can also be written as

$$
n>\frac{\ln (b-a)-\ln (\varepsilon)}{\ln (2)}-1
$$

Which means for a specific $\varepsilon$, the number of iterations depends on the interval $[a, b]$. Consequently, the computational complexity of the method depends on the interval $[a, b]$. In obtaining the computational complexity of an arbitrary characteristic equation finding the proper $a$ and $b$ is impossible because one cannot predict the changes of the interval $[a, b]$ when the order of the equation grows. In many cases, by increasing the order of the equation, the interval $[a, b]$ grows too with an indefinite rate. This way, not only the number of iterations grows, but also finding the rate of the growth becomes impossible.

However, when we consider near-regular graphs, the interval $[a, b]$ is limited and measurable. In a near-regular graph the regular part is the dominant part regarding the definition of nearregular graphs. Moreover, the eigenvalues of the main structure are close to those of the corresponding regular part. Consequently, for finding the domain of the problem (the interval $[a, b])$, one should obtain the domain of the regular structure, showing the lower and upper bounds are independent of the order of the equation. In graph product theory, the regular forms are presented in Kronecker forms, (Eq.2).

Since the matrices $\mathbf{A}_{1}$ and $\mathbf{A}_{2}$ represent the number of sectors in a graph, in comparison with the unchanged matrices $\mathbf{B}_{1}$ and $\mathbf{B}_{2}$ which represent the dimension of each sector, the matri- 
ces $\mathbf{A}_{1}$ and $\mathbf{A}_{2}$ are the basic matrices and their dimension will increase when the graph $\mathbf{M}$ expands.

Therefore, the matrices $\mathbf{A}_{1}$ and $\mathbf{A}_{2}$ control the bounds of the eigenvalues. Consider the Fig. 3

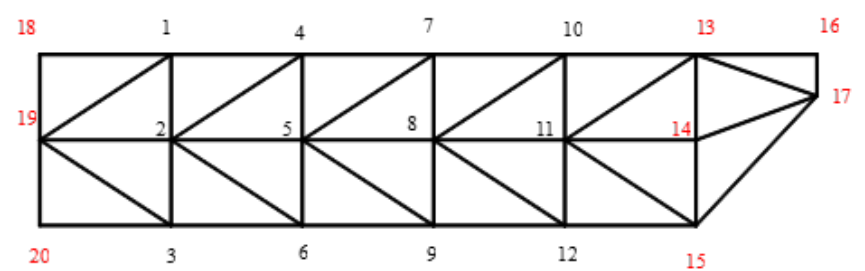

Fig. 3. A near-regular structure with a suitable ordering

In such a near-regular structure the black nodes form the regular matrix $\mathbf{M}_{11}$ as

$$
\begin{aligned}
& \mathbf{M}_{11}=\mathbf{F}(\mathbf{C}, \mathbf{B}, \mathbf{C})=\mathbf{I} \otimes \mathbf{C}+\mathbf{T} \otimes \mathbf{B} \\
& \mathbf{T}=\mathbf{F}(0,1,0)
\end{aligned}
$$

The dimension of the matrices $\mathbf{I}$ and $\mathbf{T}$ represent the number of regular sectors that is equal to 4 in this example and the matrices $\mathbf{B}$ and $\mathbf{C}$ represent the number of nodes in each sector that is equal to 3 here. When the graph shown in the Fig. 3 grows, the number of sectors grows (I and $\mathbf{T}$ ), while the number of nodes in each sector does not change (B and $\mathbf{C}$ ). Thus the basic matrices $\mathbf{A}_{1}$ and $\mathbf{A}_{2}$ control the bounds and computational complexity.

The known basic matrices in graph products include two groups: 1) tri-diagonal matrices 2) Circulant matrices

Consider the following tridiagonal matrix for the first group:

$$
Z_{n}=\left[\begin{array}{ccccccc}
-\alpha+b & c & 0 & 0 & \ldots & 0 & 0 \\
a & b & c & 0 & \ldots & 0 & 0 \\
0 & a & b & c & \ldots & 0 & 0 \\
\ldots & \ldots & \ldots & \ldots & \ldots & \ldots & \ldots \\
0 & 0 & 0 & 0 & \ldots & a & -\beta+b
\end{array}\right]_{n \times n}
$$

The eigenvalues of such a matrix is presented in [17] using the following equation:

$$
\lambda=b+2 \sqrt{a c} \cos \theta \theta \neq m \pi, m \epsilon Z
$$

Where $\theta$ is calculated as follows:

$a c \sin (n+1) \theta+(\alpha+\beta) \sqrt{a c} \sin n \theta+\alpha \beta \sin (n-1) \theta=0$

In a regular graph $\alpha$ and $\beta$ are equal to zero and $a=c$, then Eq. [19] is converted to

$$
a^{2} \sin (n+1) \theta=0
$$

And Eq. 18 changes to the following:

$$
\lambda=b+2 a \cos \theta \theta \neq m \pi, m \epsilon Z
$$

Consequently, in a regular graph when the number of sectors increases, according to Eq. 21] the lower and upper bounds of the eigenvalues does not change. This is because $n$ in Eq. 20. influence only the $\cos \theta$ in Eq. (21) which is always in the interval $[-1,1]$. Therefore the lower and upper bounds of the eigenvalues in Eq. (21) are found. Using Eq. (3), the eigenvalues for a block tri-diagonal matrix is obtained.

Now, we consider the second basic group so-called circulant matrices:

$$
P=\left[\begin{array}{ccccc}
0 & 1 & 0 & \ldots & 0 \\
0 & 0 & 1 & \ldots & 0 \\
\vdots & \vdots & \vdots & \vdots & \vdots \\
0 & 0 & 0 & \ldots & 1 \\
1 & 0 & 0 & \ldots & 0
\end{array}\right]_{n \times n}
$$

The eigenvaluse of such a matrix are obtained via solving

$$
\operatorname{det}(P-\lambda I)=\lambda^{n}-1=0
$$

The lower and upper bounds of the $\lambda$ in Eq. 23) are simply obtained in the interval $[-1,1]$ for real values. These bounds are independent of the magnitude of the n. Again, using Eq. (3) the eigenvalues of a three-block diagonal matrix are found.

Now, according to Eq. (15), since the value of $\ln (b-a)$ is measurable and limited, the number of iterations are determined for a regular graph with any arbitrary dimension. Therefore, the computational complexity of the iteration method is related to the number of roots that is $n$ roots in a graph of dimension $n$ and consequently the complexity of the iteration step is $\mathbf{O}(n)$. It was mentioned that regular graphs hold the relationship $\mathbf{A}_{1} \mathbf{A}_{2}=\mathbf{A}_{2} \mathbf{A}_{1}$. These matrices are tri-block diagonal matrices holding the patterns of $\mathbf{G}_{n}\left(\mathbf{A}_{m} \mathbf{B}_{m} \mathbf{C}_{m}\right)$ and $\mathbf{F}_{n}\left(\mathbf{A}_{m} \mathbf{B}_{m} \mathbf{C}_{m}\right)$ that decompose to $\mathrm{n}$ blocks of dimension $m$. Therefore, only $\mathrm{n}$ times the eigenvalues of matrices of dimension $m$ should be obtained. Then, the inverse of the matrix $\mathbf{M}_{11}-\lambda \mathbf{I}_{n}$ is obtained using the eigenvalues. It was mentioned as the dimension of the matrix $\mathbf{M}_{11}-\lambda \mathbf{I}_{n}$ approach to infinity, $m$ (the number of nodes in a sector) does not change and only $n$ as the number of sectors grows. As a result, the complexity of inverting the matrix $\mathbf{M}_{11}-\lambda \mathbf{I}_{n}$ is also $\mathbf{O}(n)$. Again, consider the main equation as

$$
\operatorname{det}\left(\mathbf{M}_{22}-\lambda \mathbf{I}_{m}-\mathbf{M}_{21}\left(\mathbf{M}_{11}-\lambda \mathbf{I}_{n}\right)^{-\mathbf{1}} \mathbf{M}_{12}\right)=0
$$

After obtaining the value of $\left(\mathbf{M}_{11}-\lambda \mathbf{I}_{n}\right)^{-\mathbf{1}}$ for a specific $\lambda$ in an iteration the complexity of the term $\mathbf{M}_{21}\left(\mathbf{M}_{11}-\lambda \mathbf{I}_{n}\right)^{\mathbf{- 1}} \mathbf{M}_{12}$ should be obtained. This is multiplication of three matrices in which the computational complexity is $\mathbf{O}\left(m n^{2}\right)$ Since $\mathrm{m}$ is limited, the computational complexity is $\mathbf{O}\left(n^{2}\right)$ which is the largest and dominant complexity in solving this equation. Thus, an efficient complexity is gained using the present method for the near-regular graphs. It is less complicated than other efficient known methods such as the method which uses a combination of Householder and QR methods with the complexity of $\mathbf{O}\left(n^{3}\right)$

Now, the present method is outlined in the following steps: 
1 Ordering the near-regular graph so that first the regular part is numbered and then the irregular part.

2 Forming the Laplacian (stiffness) matrix for the graph (structure). The matrix will have the pattern of the two-block matrix $\mathbf{M}$ because of the mentioned ordering.

3 Forming Eq. (9) as the main equation.

4 Forming a Laplacian (stiffness) matrix named $\mathbf{M}^{*}$ of the dimension $n+m$ (similar to the dimension of matrix $\mathbf{M}$ ) but with the pattern of matrix $\mathbf{M}_{11}$.In other words, forming a regular matrix corresponding to the near-regular matrix with the same dimension (see Eq. (12)).

5 Finding the eigenvalues of the regular matrix $\mathbf{M}^{*}$ through a quick solution using Eq. (3). These eigenvalues are used as the suitable initial guesses as they are close to the eigenvaluse of the near-regular graph. The eigenvalues are in an interval $[a, b]$ which is independent of the dimension of the matrix $\mathbf{M}^{*}$.

6 Solving Eq. (9) through an iterative bisection method without forming the characteristic equation. The eigenvalues found in step 5 are used as initial guesses in this step. Applying the iterative method in advance makes the opportunity of solving $\left(\mathbf{M}_{11}-\lambda \mathbf{I}_{n}\right)^{-\mathbf{1}}$ quickly using Eq. (10), decreasing the complexity of the method.

Previously, the inverse of the stiffness matrix of near-regular structures was found. The inverted matrix can be calculated for a near-regular structure convertible to a regular form. These kinds of near-regular structures were presented in the references [8,9].

In Eq. (9), the ordering is performed so that the matrix $\mathbf{M}_{11}$ $\lambda \mathbf{I}_{n}$ is regular and the condition $\mathbf{A}_{1} \mathbf{A}_{2}=\mathbf{A}_{2} \mathbf{A}_{1}$ holds. Therefore, inverting this matrix using the eigenpairs and Eq. 10 is possible. However, since the stiffness matrix of near-regular structures can be efficiently inverted [8,9], ordering of the matrix can also be performed so that the block $\mathbf{M}_{11}$ is a near-regular matrix. Thus, the matrix $\mathbf{M}_{11}-\lambda \mathbf{I}_{n}$ is swiftly inverted while the condition $\mathbf{A}_{1} \mathbf{A}_{2}=\mathbf{A}_{2} \mathbf{A}_{1}$ does not hold. This way the present solution gets more generalized where the matrix $\mathbf{M}_{11}$ can be both regular and near-regular. The efficiency of the method is shown using some examples.

\section{A Simple Illustrative Example}

For the truss shown in the Fig. 4, the eigenvalues of the stiffness matrix are calculated. For all members the elastic modulus is considered as $210 \mathrm{kN} / \mathrm{mm}^{2}$ and the cross section areas are taken as $15 \mathrm{~cm}^{2}$.

Based on the present algorithm, first a proper ordering is performed. According to Fig. 5, the truss is composed of regular and irregular parts that are shown in green and red colors, respectively.

Ordering of the regular part is performed sector by sector as it is shown in Fig. 5 In this structure each five nodes form a sector, for example the first sector is composed of the nodes 1,2 ,

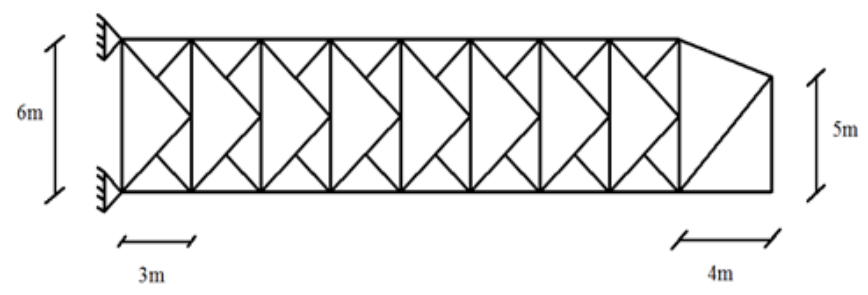

Fig. 4. The truss under study

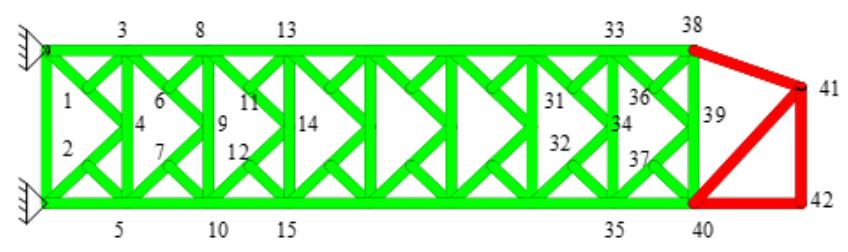

Fig. 5. The truss composed of regular and irregular parts with a suitable ordering

3, 4 and 5. Thus, the matrix $\mathbf{M}_{11}$ contains 7 blocks (each sector represents a block) of dimension 10 (each sector consists of 5 nodes with each node having two degrees of freedom). The last regular sector does not belong to the matrix $\mathbf{M}_{11}$ since it has 2 nodes in common with the irregular part (the nodes 38 and 40) that causes this sector not to follow the pattern of other regular sectors. Consequently, the sector number 8 is considered as a part of the matrix $\mathbf{M}_{22}$ as well as the irregular part shown in the red color. The matrix $\mathbf{M}_{22}$ contains 7 nodes and is of dimension 14. Using this partitioning, the reduced stiffness matrix will have the following form:

$$
\mathbf{M}=\left[\begin{array}{ll}
\mathbf{M}_{11} & \mathbf{M}_{12} \\
\mathbf{M}_{21} & \mathbf{M}_{22}
\end{array}\right]_{84 \times 84}
$$

where $\mathbf{M}_{12}, \mathbf{M}_{21}$ and $\mathbf{M}_{22}$ are matrices of dimensions $70 \times 14$, $14 \times 70$ and $14 \times 14$, respectively, and

$$
\mathbf{M}_{11}=\mathbf{F}_{70 \times 70}(\mathbf{C}, \mathbf{B}, \mathbf{C})=\mathbf{I} \otimes \mathbf{C}+\mathbf{T} \otimes \mathbf{B}
$$

where $\mathbf{B}$ and $\mathbf{C}$ are matrices of the dimension 10.

For finding the initial guesses and the interval $[a, b]$ the matrix $\mathbf{M}^{*}$ should be formed. It was mentioned that this matrix should have dimension identical to that of the matrix M. But, since the dimension of the matrix $\mathbf{M}$ is 84 , the matrix $\mathbf{M}^{*}$ cannot be formed because it is composed of blocks of dimension 10 . In other words, this matrix just can have dimensions of the multiples of 10 such as 80 or 90 . To overcome the problem, 6 rows and columns filled with zeros are added to the matrix $M$ to get the dimension 90. These zeros do not affect the eigenvalues of the matrix $\mathbf{M}$ and only add 6 additional zero eigenvalues to the final answer. This new matrix is named $\mathbf{M}^{\prime}$

$$
\mathbf{M}^{\prime}=\left[\begin{array}{ccc}
\mathbf{M} & \vdots & \mathbf{0}_{84 \times 6} \\
\cdots & \cdots \\
\mathbf{0}_{6 \times 84} & \vdots & \mathbf{0}_{6 \times 6}
\end{array}\right]_{90 \times 90}
$$


The matrix $\mathbf{M}^{*}$ of the dimension 90 (9 blocks of the dimension 10) is formed as:

$$
\mathbf{M}^{*}=\mathbf{F}(\mathbf{C}, \mathbf{B}, \mathbf{C})=\mathbf{I} \otimes \mathbf{C}+\mathbf{T} \otimes \mathbf{B}
$$

In fact the matrix $\mathbf{M}^{*}$ is the stiffness matrix of the regular structure of Fig. 6 .

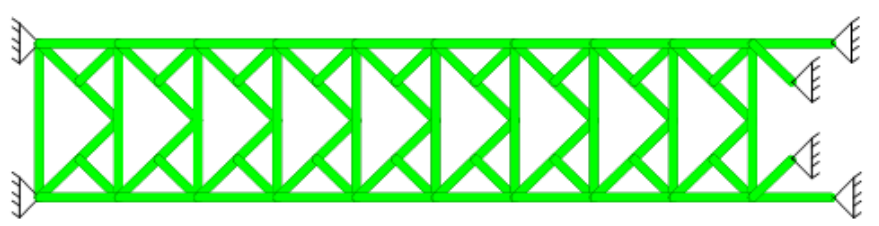

Fig. 6. A regular structure used for appropriate initial guesses

The supports in the right side are chosen so that the last sector is similar to the other sectors.

Now using Eq. (3), the eigenvalues of the matrix $\mathbf{M}^{*}$ are obtained. There are 90 eigevalues for the matrix $\mathbf{M}^{*}$. Typical eigenvalues $\lambda_{11}$ to $\lambda_{20}$ are written in the following:

$$
\lambda_{\mathbf{M}^{*}}=10^{6}[1.7329,1.8604,2.0003,2.0649,
$$

$2.4029,2.4228,2.9563,3.4094,3.5748,3.9336]$

Since for the matrix $\mathbf{M}^{*}, \lambda_{\min }=\lambda_{1}=0.2951 \times 10^{6}$ and $\lambda_{\max }=\lambda_{90}=63.461 \times 10^{6}$ and regarding the six zero eigenvalues added to the main structure, the interval used in the bisection method is chosen as [0 $\left.65 \times 10^{6}\right]$.

The initial guesses and the interval are ready to be utilized in the bisection method. All terms in the equation

$$
\operatorname{det}\left(\mathbf{M}_{22}-\lambda \mathbf{I}_{m}-\mathbf{M}_{21}\left(\mathbf{M}_{11}-\lambda \mathbf{I}_{n}\right)^{-\mathbf{1}} \mathbf{M}_{12}\right)=0
$$

were calculated. Now, the equation is solved in each step using the bisection method. The eigenvalues $\lambda_{11}$ to $\lambda_{20}$ are found as

$$
\begin{aligned}
& \lambda_{\mathbf{M}}=10^{6}[1.5934,1.8151,1.9138,2.1311, \\
& 2.2158,2.7474,3.1877,3.3628,3.5226,3.9020]
\end{aligned}
$$

It can be observed that $\lambda_{\mathbf{M}}^{*}$ is an efficient initial guess for $\lambda_{\mathbf{M}}$. In order to obtain more precise answers, one can use Rayleigh-Ritz relationship by means of which having the approximate eigenvalues and eigenvectors, one can get closer to the eigenvalues and eigenvectors of the main problem.

\section{Numerical Examples}

In this section different examples are studied to show the efficiency of the present method.

Example : In this example the frequencies of the free vibration of the double layer grid, shown in the Fig. 7 are calculated. A real case of such a grid can be seen in Fig. 8 . For all members the elastic modulus is taken as $200 \mathrm{kN} / \mathrm{mm}^{2}$ and the cross section areas are $10 \mathrm{~cm}^{2}$. The mass is equal to $7.8 \mathrm{~kg}$ for the vertical and horizontal members and $11.0 \mathrm{~kg}$ for the diagonal ones.
The plan of the structure and its lateral view can be seen in the Figs. 9and 10 respectively.

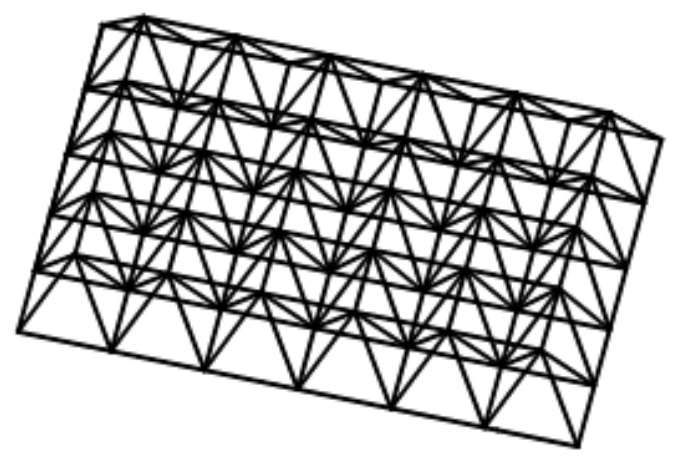

Fig. 7. A double layer grid with near regular pattern

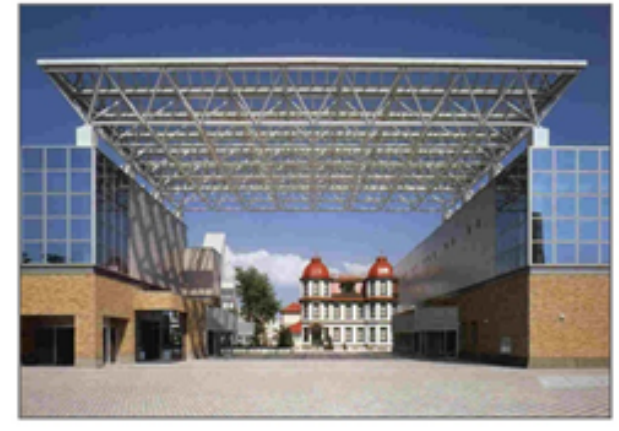

Fig. 8. A real double layer grid

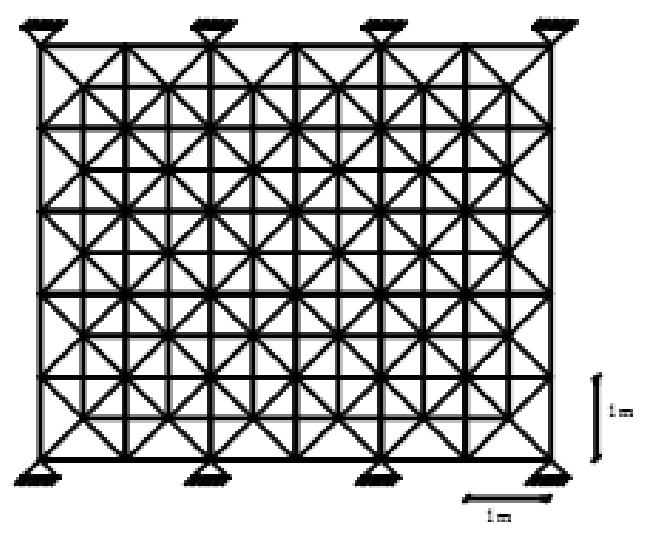

Fig. 9. The plan of the grid

To find the frequencies of the structure, the following equation should be solved:

$$
\left(\mathbf{K}-\omega_{i}^{2} \mathbf{M}\right)\{\emptyset\}_{i}=\{\mathbf{0}\}
$$

Multiplying the two sides by $\mathbf{M}^{\mathbf{- 1}}$, we will have

$$
\left(\mathbf{M}^{-\mathbf{1}} \mathbf{K}-\omega_{i}^{2} \mathbf{I}\right)\{\emptyset\}_{i}=\{\mathbf{0}\}
$$

And then

$$
\operatorname{det}\left(\mathbf{M}^{-1} \mathbf{K}-\omega_{i}^{2} \mathbf{I}\right)=\mathbf{0}
$$




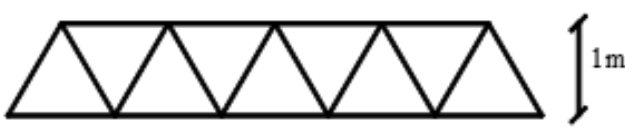

Fig. 10. Lateral view of the grid

The structure is geometrically regular. However, it is considered as a near-regular structure mathematically using a suitable nodal ordering. In this paper, a graph or structure is considered as regular if the pattern of its Laplacian matrix or stiffness matrix follows graph product rules. A graph or structure with just a regular geometry is not considered as regular. The structure is composed of a central regular part shown in green and an irregular part shown in red, Fig. 11. Using a proper ordering and considering $\mathbf{M}^{\mathbf{- 1}} \mathbf{K}=\mathbf{S}$, the matrix $\mathbf{S}$ of the dimension 192 with the following pattern is obtained:

$$
\mathbf{S}=\left[\begin{array}{ll}
\mathbf{S}_{11} & \mathbf{S}_{12} \\
\mathbf{S}_{21} & \mathbf{S}_{22}
\end{array}\right]
$$

where $\mathbf{S}_{11}$ is the block of the regular green part, and $\mathbf{S}_{22}$ corresponds to the block of the irregular red part.

Using the blocks of the matrix , the main equation is formed

$$
\operatorname{det}\left(\mathbf{S}_{22}-\omega^{2} \mathbf{I}_{m}-\mathbf{S}_{21}\left(\mathbf{S}_{11}-\omega^{2} \mathbf{I}_{n}\right)^{-\mathbf{1}} \mathbf{S}_{12}\right)=0
$$

The solution of this equation using the present algorithm results in finding the values of $\omega$. The first 10 frequencies are obtained as

$$
\omega=10^{4}[0.8791,1.1253,1.3324,1.6811,1.9371 \text {, }
$$

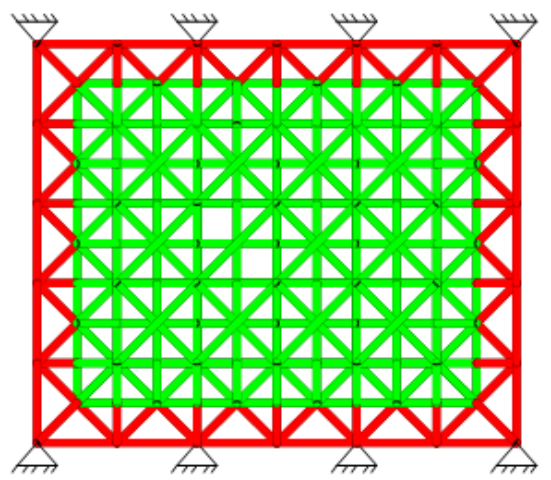

Fig. 11. The regular and irregular parts of a double layer grid.

Example : An important practical application of the present method is in the analysis of structures with constructional imperfection. Consider the structure shown in Fig. 12 Because of constructional imperfection the nodes 31 and 35 are not in the right place as shown in Fig. 13 . The amount of their deviation is seen in Fig. 13. For all members, the elastic modulus is taken as $210 \mathrm{kN} / \mathrm{mm}^{2}$ and the cross section areas are $15 \mathrm{~cm}^{2}$. The specific gravity of steel is equal to $7800 \mathrm{~kg} / \mathrm{m}^{3}$. The frequencies of the real structure are found.

The frequencies of the regular ideal structure shown in Fig. 12 are swiftly found using graph products. However, due to the constructional imperfection, the real structure changes into a near-regular one composed of regular and irregular parts as shown in Fig. 14

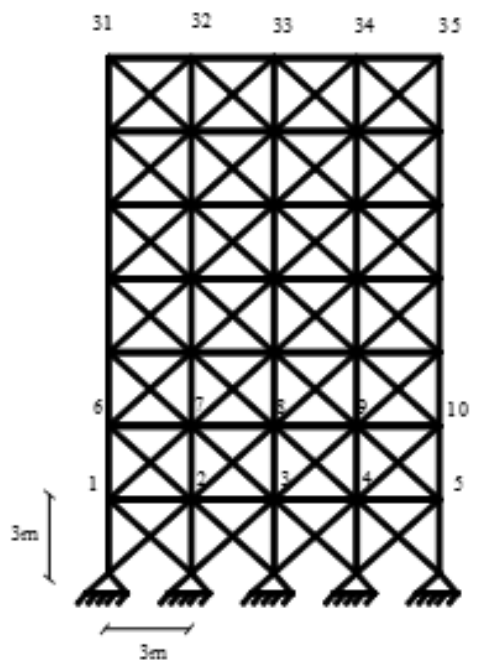

Fig. 12. The ideal form of the truss under study

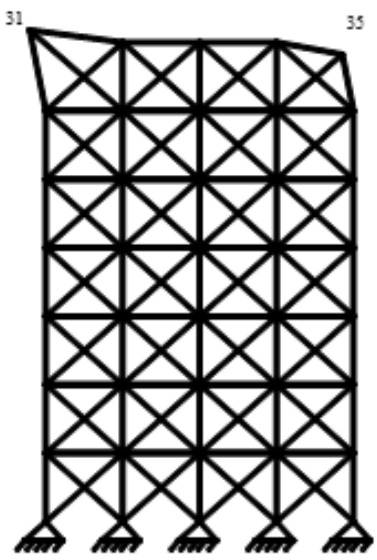

Fig. 13. The real form of the truss including constructional imperfection

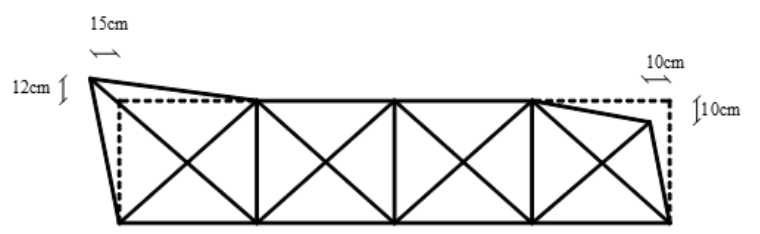

Fig. 14. Constructional imperfection of the last story

To solve the real structure, the frequencies of the regular ideal structure can be used as an initial guess. The main equation is written as

$$
\operatorname{det}\left(\mathbf{S}_{22}-\omega^{2} \mathbf{I}_{m}-\mathbf{S}_{21}\left(\mathbf{S}_{11}-\omega^{2} \mathbf{I}_{n}\right)^{-\mathbf{1}} \mathbf{S}_{12}\right)=0
$$




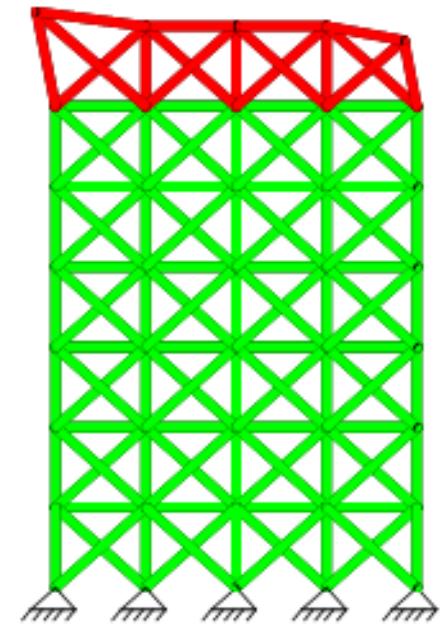

Fig. 15. A structure composed of regular and irregular parts

where the matrix $\mathbf{S}_{11}$ with dimension 50, represents the green regular part, and $\mathbf{S}_{22}$ with dimension 20 , represents the red irregular part. Using Eq. (3), the frequencies of the ideal structure are calculated. The frequencies $\omega_{61}$ to $\omega_{70}$ are obtained as

$$
\begin{aligned}
& \omega=10^{4}[15.3324,15.7053,16.1053,16.4158,16.7371, \\
& 17.0850,17.3017,17.5989,17.7348,17.9126]
\end{aligned}
$$

Now, these frequencies are used as the initial guess in the bisection stage of the present algorithm. The frequencies $\omega_{61}$ to $\omega_{70}$ of the real structure are obtained as

$\omega=10^{4}[15.6824,15.9358,16.2489,16.5298,16.8626$,

17.2007, 17.4611, 17.7218, 17.9346, 18.1043]

\section{Concluding Remarks}

In this paper an efficient algorithm is presented to calculate the eigenvalues (frequencies of free vibration) of near-regular structures composed of regular and irregular parts. The method is a combination of both exact and iterative solutions. The regular and irregular parts get separated using a proper ordering and thus a two block matrix is obtained. This matrix is then decomposed using the rules of the partitioned block matrix and the main equation is obtained. The solution of the gained equation results in the eigenvalues. Instead of forming the characteristic equation and then applying the iterative method, the main equation is directly solved using an iterative bisection method. This direct solution provides the opportunity for applying the graph product rules for a swift solution. The efficiency of the method depends on the computational complexity of the iterative method. For an arbitrary matrix where the graph product rules do not hold and there is no effective initial guess for the iterative method, the method is as efficient as the other existing approaches. However, when near-regular structures are considered, since the graph product rules are set in the regular part and the initial guesses with limited bounds are available, the method is less complex than other approaches. The presented method is applicable to calculating the vibrational frequencies of nearregular structures, rehabilitated structures, structures with constructional imperfection and similar problems.

\section{References}

1 Kaveh A, Optimal Analysis of Structures by Concepts of Symmetry and Regularity, Springer Verlag; Wien-New York, 2013, DOI 10.1007/978-3-70911565-7

2 Law SS, Yong D, Substructure methods for structural condition assessment, Journal of Sound and Vibration, 330(15), (2011), 3606-3619, DOI 10.1016/j.jsv.2011.03.003

3 Biondi B, Muscolino G, Component-mode synthesis method for coupled continuous and FE discretized substructures, Engineering Structures, 25(4), (2003), 419-433, DOI 10.1016/S0141-0296(02)00183-9

4 Kaveh A, Beheshti S, Weighted triangular and circular graph products for configuration processing, Periodica Polytechnica Civil Engineering, 56(1), (2012), 63-71, DOI 10.3311/pp.ci.2012-1.07

5 Kaveh A, New developments in graph products and applications in structural engineering, Pollack Periodica, 7(Supplement 1), (2012), 105-117, DOI $10.1556 /$ Pollack.7.2012.S.10

6 Kaveh A, Massoudi M S, Massoudi M J, Efficient finite element analysis using graph-theoretical force method; rectangular plane stress and plane strain serendipity family elements, Periodica Polytechnica Civil Engineering, 58(1), (2014), 3-22, DOI 10.3311/PPci.7405

7 Fang $\mathbf{H}$, Wang $\mathbf{T J}$, Chen $\mathbf{X}$, Model updating of lattice structures: A substructure energy approach, Mechanical Systems and Signal Processing, 25(5), (2011), 1469-1484, DOI 10.1016/j.ymssp.2011.01.002

8 Shojaei I, Kaveh A, Rahami H, Analysis of structures convertible to repeated structures using graph products, Computers \& Structures, 125, (2013), 153-163, DOI 10.1016/j.compstruc.2013.04.018

9 Rahami H, Kaveh A, Shojaei I, Gholipour Y, Analysis of Irregular Structures Composed of Regular and Irregular Parts Using Graph Products, Journal of Computing in Civil Engineering, 28(4), (2014), 04014016, DOI 10.1061/(ASCE)CP.1943-5487.0000375

10 Shojaei I, Rahami H, Kaveh A, Efficient finite element solution of regular and near-regular systems using graph products, Acta Mechanica, 226(7), (2015), 2393-2405, DOI 10.1007/s00707-015-1337-z

11 Kaveh A, Shojaei I, Rahami H, New developments in the optimal analysis of regular and near-regular structures: decomposition, graph products, force method, Acta Mechanica, 226(3), (2015), 665-681, DOI 10.1007/s00707014-1194-1

12 Kaveh A, Rahami H, Mehanpour H, Static and modal analyses of structures with different repeated patterns, Advances in Engineering Software, 51, (2012), 1-9, DOI 10.1016/j.advengsoft.2012.04.007

13 Kaveh A, Rahami H, Block diagonalization of adjacency and Laplacian matrices for graph product; applications in structural mechanics, International Journal for Numerical Methods in Engineering, 68(1), (2006), 33-63, DOI $10.1002 / \mathrm{nme} .1696$

14 Kaveh A, Rahami H, Compound matrix block diagonalization for efficient solution of eigenproblems in structural mechanics, Acta Mechanica, 188(34), (2007), 155-166, DOI 10.1007/s00707-006-0364-1

15 Kaveh A, Rahami $\mathbf{H}$, An efficient analysis of repetitive structures generated by graph products, International Journal for Numerical Methods in Engineering, 84(1), (2010), 108-126, DOI 10.1002/nme.2893

16 Pothen A, Simon HD, Liou K-P, Partitioning Sparse Matrices with Eigenvectors of Graphs, SIAM Journal on Matrix Analysis and Applications, 11(3), (1990), 430-452, DOI $10.1137 / 0611030$

17 Yueh W-C., Eigenvalues of several tri-diagonal matrices, Applied Mathematics, 5, (2005), 66-74. 